\title{
WAY AHEAD FOR HEALTHCARE SECTOR POST-COVID-19
}

The COVID pandemic has brought to forefront the challenges of the public health system. India needs well-equipped government hospitals and not grand buildings, which will serve no public purpose. In our country there is a gross mismatch between the healthcare resource distribution and the needy clientele. With less than 1 physician per 1000 population, India is well behind its peer countries. It needs an additional 3.6 million hospital beds to reach the recommended capacity. Only about $27 \%$ of India's population is covered around any form of health insurance and the out of pocket expenditure on health is $62.4 \%$ in India as compared to the world average of $18.2 \%$. Two-thirds of hospital beds in India and almost $80 \%$ of available ventilator-equipped ICU beds are in private hospitals. At $3.6 \%$ of GDP, India's overall health spending is among the lowest compared with peer and advanced economies. Of this, government spending on health is less than $1 \%$. Alarmingly, out-of-pocket health expenditure for households is extraordinarily high in India. About $65 \%$ of all health expenditure in India (approx $2.5 \%$ of GDP) is borne privately by households. India could find itself in an acute health crisis over the next few months and the direct cause of it will not be the SARS-CoV-2 virus. Hundreds of thousands of children might already have missed vital immunizations. The shutdown of the economy has inflicted unbearable social and economic costs. An estimated 122 million jobs in the formal and informal sectors have been lost. The informal sector, which employs $90 \%$ of the workforce and the MSMEs, are worst hit.

In the past 10 years, there is a shift in disease pattern, India's growing noncommunicable disease — cardiovascular ailments, cancer, respiratory diseases, diabetes, and mental health conditions are seeking most attention, both resource wise and financially. While containing the surge of COVID-19, we will also have to be on alert for other contagions, which assume menacing proportions during the summer in various states.

\section{Challenges}

- Currently routine healthcare has taken a back seat due to disrupted hospital services, primary care services are being provided by government and private providers. Post-Covid-19 prevalence of virus in community is going to stay. This will result in reduced foot falls and elective procedures. Health expenditure will get pushed back in the list of priorities.The fear of catastrophic expenditure becoming a norm cannot be ruled out.

- The epidemic has compromised the Ayushman Bharat Pradhan Mantri Jan Arogya Yojana's (PMJAY) ability to reach out to critically ill patients - including those afflicted with cancer and below the poverty line. Hospital industry is going through trying times. There has been a deep fall in OPD volumes and planned surgeries.

- Financial recession in hospitals/ Reduced Revenue will be due to lockdown hospitals are witnessing a drop in both domestic and international patient footfalls and elective surgeries, resulting in occupancy levels to fall to a mere $40 \%$ by late-March vis-à-vis preCOVID occupancy levels of $\sim 65-70 \%$ and is expected to reduce further.

- There is a huge liquidity crisis due to revenue loss and slow reimbursements (CGHS, ECHS, Insurance).

Way ahead: The support that government can provide to revive the healthsector and keep it viable for facing more challenges are as under:

- Facilitating Finanacial Improvement:

- Government should provide a tax holiday for at least two years to start with to all health sector private hospitals to boost up their financial position.

- Government should give two-year moratorium on all working capital, principal, interest payments on loans and overdrafts, bringing in liquidity and enabling business continuity.

- A waiver of GST on input costs and services for 2 years.

- Twenty percent rebate on the current commercial rates of power and water rates currently being paid by hospitals, diagnostics centers, pathology labs and other healthcare service providers to ensure sustenance of business.

- Subsidy of salary and contributions toward government funds such as ESIC and PF

- Surge Capacity Building

- It is time to invest in Invest in Public Health and increase preparedness for new emerging health threats.

- We have to create rapid re-tasking models especially at lower skill levels in health system.

- Non-healthcare volunteer personnel can be trained and maintained as pool for re-tasking (Like a reserve force). This will allow up scaling of lower level health manpower for higher role. E.g. ANMs can be re-tasked for independent nursing duties up to a certain level

- Concept of RESERVISTS implemented for al healthcare personnel trained at public cost.

- Identifying existing infrastructure that can be re-appropriated on demand is highly essential

- Mandating Health as Social Security.

- Comprehensive medical and life insurance benefits for all employees in health sector.

- Advance payment for patients being treated under various government schemes such as Ayushman Bharat, CGHS, etc.

- Revision and realistic rates for various procedures be put in place for various schemes.

- PHCs should be made attractive to doctors by providing incentives and making rural service mandatory for medical students. 
- Developing MAKE IN INDIA Resources.

- Right opportunity for Pharma and Medical Device Industry to encash by exporting Drugs and Medical Devices to large number of countries who need these support.

- More AMTZ like facilities need to be opened across the country to promote Make in India thereby making india a power to reckon with in Manufacturing hub.

- Good opportunity to attract FDI in manufacturing sector as many countries want to withdraw from China.

- Right time to explore developing alternative sources of getting raw material for manufacturing drugs rather than depending on China alone.

- Encourage entrepreneurship - Providing tax incentives, rebate on factors and facilitate industrial hubs for medical equipment. Provisioning of R\&D support to the maximum.

- MSMEs need to be given a special push and encouragement for enhancing the production, increasing the product line and turnover capacities in a phased manner across the country by not loosing the status MSME.

- MSMEs only can absorb the semi skilled and low skilled wage earners. To enhance output of the same units the skill development of trade specific can be imparted at Industries as per registration by industry and its demand at nominal fee. This would reduce crowd aggregation at a general place for skill development training and can reduce the Covid-19 infection spreading in the present scenario.

- Innovation and Standard Based Care

- Standardized clinical processes, which were not being accepted due to reflex defence of "clinical autonomy", will be the primary basis for excellence. This needs to change. Standard based care is the new norm.

- Especially useful for handling ethical dilemmas - if and when resources reach their limits, such as rationing ventilators.

- It should put out standard treatment guidelines for public and private providers, frame a patients charter of rights, engage with professional associations and civil society, and establish a regular audit system.

- The government's National Innovation Council, which is mandated to provide a platform for collaboration amongst healthcare domain experts, stakeholders and key participants, should encourage a culture of innovation in India and help develop policy on innovations.

- Protecting Workforce: Workforce needs to be protected against occupational hazards and they should be properly equipped. Besides short-term motivating events such as clapping, fly-pasts, flower-shower, etc. long-term sustained motivation in terms of improved pay, facilities, etc. should be ensured.

- Redefining Health Architecture: Need to encourage nature integrated and ventilation-based constructions rather completely climate controlled structures. In Covid-19, even though an infection spreads by contact and air-borne droplets, the same failed to infect individuals in open spaces and high ventilation areas. Hence the planning and designing of Health facilities may need a fresh look. Brown field project development and retrofitting to suit the existing demand of ventilation and air circulation will be important

Shakti Kumar Gupta Medical Superintendent All India Institute of Medical Sciences Dr RP Centre for Ophthalmic Sciences New Delhi, India Editor-in-Chief, IJRFHHA

Maj Gen Sunil Kant, VSM MG (Med), EC Editor-in-Chief, IJRFHHA 